\title{
The efficacy of cryotherapy in one case of retinal vasoproliferative tumor.
}

\author{
Tatianne Fernandes Duarte ${ }^{1 *}$, Jacques Ramos Houly ${ }^{1}$, Frederico Gustavo Telles e Souza ${ }^{2}$ \\ ${ }^{1}$ Retina and Vitreous Department, Fundação Hilton Rocha, Minas Gerais, Brazil \\ ${ }^{2}$ Retina and Vitreous Department, Santa Casa Olhos de Montes Claros, Minas Gerais, Brazil
}

\begin{abstract}
Retinal vasoproliferative tumor (VPT) is uncommon retinal lesion, classified as primary (idiopathic) or secondary. Retinal VPT has a predilection for the peripheral inferior temporal quadrant of the retina and may be unilateral or bilateral. It can lead to decrease vision due to intraretinal or subretinal exudation, neovascularization, retinal detachment, vitreous hemorrhage, preretinal fibrosis, macular edema and epiretinal membrane formation. Different modalities have been used to treat this tumor including clinical observation, pars plana vitrectomy, cryotherapy, laser photocoagulation, brachytherapy and radiotherapy. The authors describe a case of idiopathic retinal VPT associated with extensive serous retinal detachment and point out the importance of diagnosis and the effectiveness of cryotherapy.
\end{abstract}

Keywords: Retinal vasoproliferative tumor, Cryotherapy, Serous retinal detachment.

Accepted on August 28, 2018

\section{Introduction}

Retinal vasoproliferative tumor (VPT) may be primary $(74 \%)$ or secondary $(26 \%)$ [1]. Primary tumor is usually solitary lesion, in contrast to secondary tumor, that is often multiple and occur in association with other ocular conditions such as, intermediate uveitis, retinitis pigmentosa, Coats disease, neurofibromatosis, retinopathy of prematurity and familial exudative vitreoretinopathy $[1,2]$.

These tumors appear clinically as a yellow-red retinal mass often associated with lipoproteinaceous exudation. Fluorescein angiography (FA) shows early filling through a normal-caliber or slightly dilated retinal feeding arteriole and diffuse leakage into the mass or surrounding subretinal or preretinal space [3].

Retinal VPT can be differentiated from capillary hemangiomas associated with Von Hippel-Lindau disease because of the absence of markedly dilated and tortuous feeder vessels, which are characteristic of the latter condition. Furthermore, patients with retinal VPT do not have a positive family history or any systemic condition, unlike patients with Von Hippel-Lindau disease [1].

This case report describes a patient with retinal VPT associated with serous retinal detachment treated successfully by cryotherapy.

\section{Case Report}

A 56-year-old male presented to the Fundação Hilton Rocha, Brazil, with complaint of decreased visual acuity in the left eye (OS)-his single eye. The patient had previous history of vitreous hemorrhage associated with rhegmatogenous retinal detachment in the right eye (OD) with vision loss. His bestcorrected visual acuity (BCVA) was hand motion in the OD and counting fingers in the OS. Anterior segment biomicroscopy and Goldmann applanation tonometry were normal. Binocular indirect ophthalmoscope was not feasible in the OD and revealed a red-orange lesion accompanied by an extensive area of hard exudates and serous retinal detachment affecting the lower quadrants of the retina and macular region in the OS (Figure 1). Fluorescein angiography showed a hyperfluorescent lesion located in the inferior and temporal quadrants in the OS, accompanied by pooling (Figure 2). Ocular ultrasound confirmed the findings ((Figure 3) preoperative B-scans on the tumor were not available). Cryotherapy of the lesion was promptly performed with complete resolution of the serous retinal detachment and improvement of the visual acuity in the OS (Figure 4). Macular region that was previously detached was completely reattached as evidenced by optical coherence tomography (Figure 5).
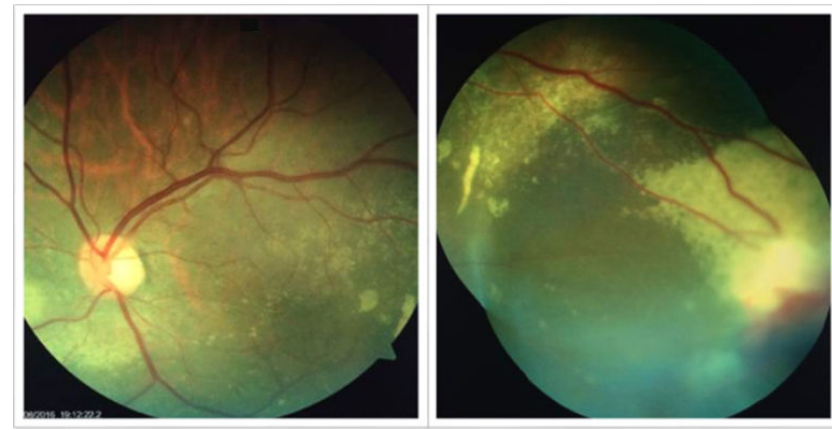

Figure 1. Fundus photography shows serous retinal detachment with involvement of the macula (left) and a red-orange tumor located in temporal and inferior quadrant of the retina associated with hard exudates. 
Citation: Tatianne FD, Jacques RH, Frederico GTS. The efficacy of cryotherapy in one case of retinal vasoproliferative tumor. $J$ Clin Ophthalmol. 2018;2(2):67-69.

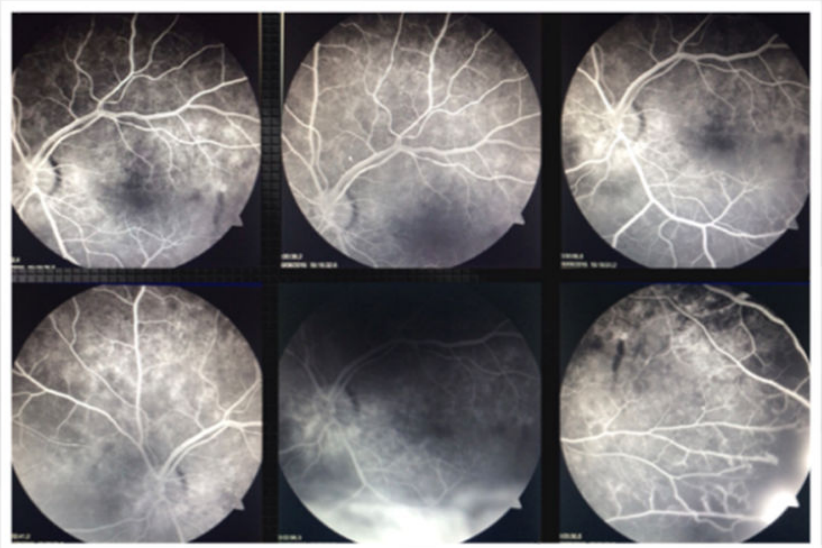

Figure 2. Fluorescein angiography reveals hyperfluorescent areas corresponding to the serous retinal detachment (middle botton) and vasoproliferative tumor (botton right).
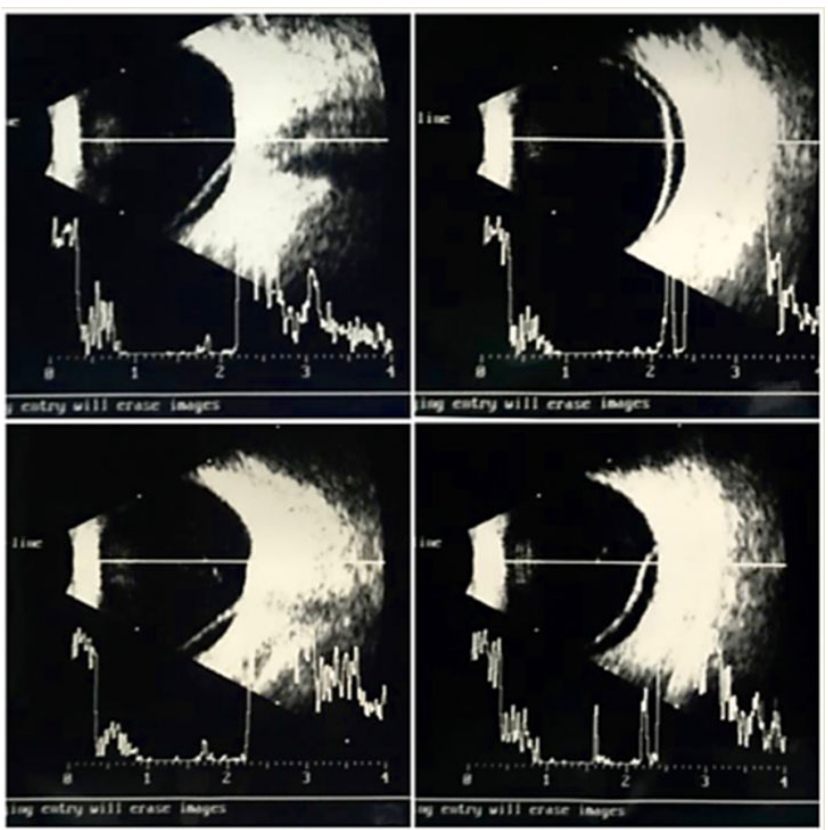

Figure 3. Ocular Ultrasound: axial and transverse B-scans show a hyperreflective membrane compatible with serous retinal detachment that reaches inferior and temporal quadrants of the retina, including the macular area.
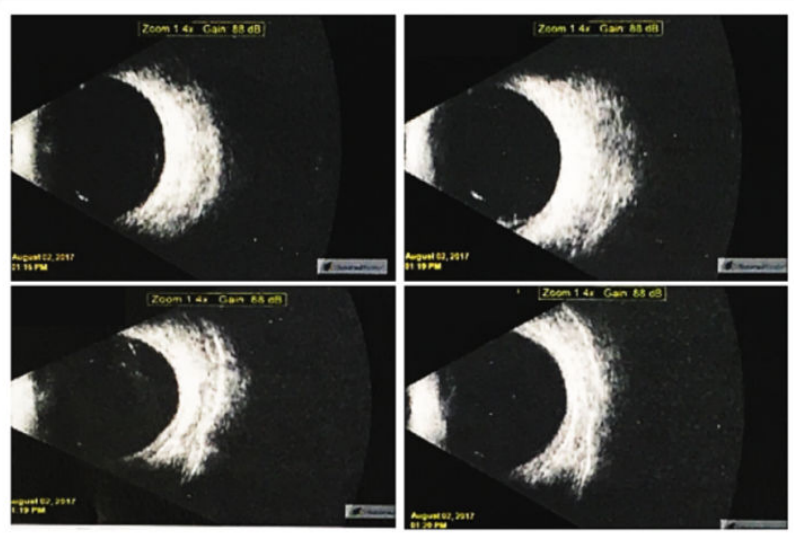

Figure 4. Ocular Ultrasound: B-Scans show complete resolution of the serous retinal detachment.

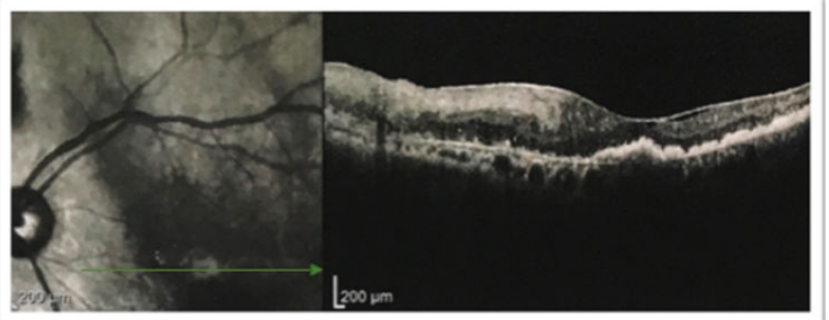

Figure 5. Spectral-domain optical coherence tomography shows an increase in the retinal thickness (juxtafoveolar area), an epiretinal membrane and changes in reflectivity within the outermost layers of the retina without subretinal fluid.

\section{Discussion}

Retinal VPT is a rare and benign disease that has capillary hemangioma as the most frequent differential diagnosis [4]. Both entities share some clinical characteristics, however, retinal capillary hemangiomas typically are associated with "twin" vessels and often represent the clinical manifestation of Von Hippel-Lindau disease. These alterations constitute benign vascular tumors, however, retinal VPT are not accompanied by dilated feeder vessels [5]. In addition, in this case report the patient had a negative screening for Von Hippel-Lindau disease. Due to all the aforementioned reasons, the clinical diagnosis was of vasoproliferative tumor.

Treatment options for retinal VPT include clinical observation, surgical procedure, cryotherapy, laser brachytherapy and radiotherapy $[2,6]$. The treatment is based on the presence of exudative retinopathy and symptoms. This tumor may be progressive and, therefore, small asymptomatic lesions should be monitored. Tumor growth, increased exudation or subretinal fluid should prompt treat to prevent debilitating visual outcomes [1,7].

In a study of 103 patients with retinal VPT, the primary treatment consisted of observation (49\%), cryotherapy (42\%), laser photocoagulation (5\%) and plaque radiotherapy [1]. Additional treatment options include photodynamic therapy (PDT), anti-angiogenic therapy, periocular or intravitreal corticosteroid administration and pars plana vitrectomy (PPV)

\section{[1].}

PDT may be used to treat both primary and secondary retinal VPT [7]. It works by producing selective damage to endothelial cells, generating localized vascular occlusion with minimal injury to the overlying retina. Some authors use PDT for retinal VPT (posterior to the equator) less than $10 \mathrm{~mm}$ in diameter $[4,6]$.

Radiotherapy is generally reserved for retinal VPT larger than $10 \mathrm{~mm}$ in diameter or those with extensive serous retinal detachment. Cohen et al. studied 30 cases of retinal VPT managed with plaque radiotherapy and found tumor regression in $97 \%$ of cases. Exudative retinal detachment was resolved in only $65 \%$ of cases [8]. On the other hand, brachytherapy can also be used for tumor that are thicker than $2.5 \mathrm{~mm}$ and lesions associated with retinal detachment $[9,10]$. 
Bevacizumab has also been used in the treatment of retinal VPT, although it is unclear, as yet, whether the initially promising result will be maintained in the long term [11].

Cryotherapy is generally used for the treatment of small peripheral retinal VPT $(<2 \mathrm{~mm})$. Larger tumor is difficult to treat with cryotherapy and more than one session is required [11]. It is worth noting that the freezing of large lesions may lead to an increase in exudation $[12,13]$.

Shields et al. refer to cryotherapy as the most frequently employed treatment modality for retinal VPT and showed a large tumor $(4.04 \mathrm{~mm})$ that was successfully treated by cryotherapy combined with anti-VEGF injection due to macular edema [1]. A similar approach was successfully employed by Rodrigues et al. who treated a $2.25 \mathrm{~mm}$ retinal VPT with cryotherapy associated to intravitreal injection of triamcinolone [14].

In this case report one session of cryotherapy was sufficient to lead to tumor regression and improvement of the visual acuity.

\section{Conclusion}

Correct diagnosis and appropriate treatment are important to halt the progression and complications of retinal VPT. In this case the retinal cryotherapy was effective in tumor regression with complete resolution of the serous retinal detachment.

\section{References}

1. Shields CL, Shields JA, Barrett J, et al. Vasoproliferative tumors of the ocular fundus. Classification and clinical manifestations in 103 patients. Arch Ophthalmol. 1995;113(5):615-23.

2. Shields JA, Shields CL, Honavar SG, et al. Clinical variations and complications of Coats disease in 150 cases: the 2000 Sanford Gifford Memorial Lecture. Am J Ophthalmol. 2001;131(5):561-71.

3. Garcia-Arumi J, Distefano LN, Fonollosa A. et al. Management of Vision-Threatening Complications of Vasoproliferative Tumors of the Retina Ophthalmic Res. 2015;54(4):34-40.

4. Blasi MA, Scupola A, Tiberti AC, et al. Photodynamic therapy for vasoproliferative retinal tumors. Retina. 2006;26(4):404-9.

5. Shields C. Retinal Capillary Hemangioma. In: Guyer D, ed. Retina-Vitreous-Macula. Philadelphia: W.B Saunders, 1999;v.2.

6. Chan RP, Lai TY. Photodynamic therapy with verteporfin for vasoproliferative tumour of the retina. Acta Ophthalmol. 2010;88:711-2.

7. Laqua $H$, Wessing A. Peripheral retinal telangiectasis in adults simulating a vascular tumor or melanoma. Ophthalmology. 1983;90:1284-91.
8. Saldanha MJ, Edrich C. Treatment of vasoproliferative tumors with photodynamic therapy. Ophthalmic Surg Lasers Imaging. 2008;39(2):143-5.

9. Cohen VM, Shields CL, Demirci H, et al. Iodine I 125 plaque radiotherapy for vasoproliferative tumors of the retina in 30 eyes. Arch Ophthalmol. 2008;126(9):1245-51.

10. Anastassiou G, Bornfeld N, Schueler AO, et al. Ruthenium-106 plaque brachytherapy for symptomatic vasoproliferative tumours of the retina. Br J Ophthalmol. 2006;90(4):447-50.

11. Kenawy N, Groenwald C, Damato B. Treatment of a vasoproliferative tumour with intravitreal bevacizumab (Avastin). Eye. 2007;21(6):893-4.

12. Marback EF, Guerra RL, Maia Junior OO, et al. Retinal vasoproliferative tumor. Arq Bras Oftalmol. 2013;76(3): 200-3.

13. Heimann $\mathrm{H}$, Bornfeld $\mathrm{N}$, Vij O, et al. Vasoproliferative tumours of the retina. Br J Ophthalmol 2000;84:1162-9.

14. Rodrigues LD, Serracarbassa LL, Rosa H, et al. Tumor vasoproliferativo associado à tuberculose ocular presumida: relato de caso. Arq Bras Oftalmol. 2007;70(3): 527-31.

\section{*Correspondence to:}

Tatianne Fernandes Duarte

42, Chumbo ST

Belo Horizonte/MG, Brazil,

Zip code: 30210-540

Phone number: (55) (31) 99330-5785,

E-mail: tatianneduarte@yahoo.com.br 